\title{
Minimax Optimal Control for Atmospheric Fly-Through Trajectories ${ }^{1}$
}

\author{
P. LU $^{2}$ AND N. X. VINH ${ }^{3}$ \\ Communicated by A. Miele
}

\begin{abstract}
Necessary conditions for minimax problems with isolated or flat maxima are presented. Some relevant properties concerning the peak heating rate and the peak deceleration during atmospheric entry are discussed. As application of the theory, the problem of minimizing the peak heating rate of a skip trajectory is solved with special emphasis on the discussion of the continuity of the lift control at the point where the maximum occurs along the trajectory.
\end{abstract}

Key Words. Chebyshev problems, minimax problems, optimal trajectories, reentry trajectories, lift modulation.

\section{Introduction}

The minimization of the peak heating rate and the minimization of the peak deceleration of a hypervelocity reentry vehicle by controlling the aerodynamic forces belong to the class of Chebyshev minimax problems. The objective of these problems is to find an optimal control $\mathbf{u}(t), t_{0} \leq t \leq t_{f}$, for the following system:

$$
\begin{aligned}
& \min \max _{t_{0} \leq t \leq t_{f}} F(\mathbf{x}(t)), \\
& \dot{\mathbf{x}}=\mathbf{f}(\mathbf{x}, \mathbf{u}), \\
& \mathbf{u}(t) \in U, \quad \forall t \in\left[t_{0}, t_{f}\right], \\
& \mathbf{x}\left(t_{0}\right)=\mathbf{x}_{0}, \\
& S\left(\mathbf{x}\left(t_{f}\right)\right)=0 .
\end{aligned}
$$

\footnotetext{
${ }^{1}$ This research was supported by the Jet Propulsion Laboratory, Contract No. 916416, with Dr. K. D. Mease as project manager.

${ }^{2}$ Research Assistant, Department of Aerospace Engineering, University of Michigan, Ann Arbor, Michigan.

${ }^{3}$ Professor of Aerospace Engineeering, University of Michigan, Ann Arbor, Michigan.
} 
In the above equations, $\mathbf{x} \in \mathbf{R}^{n}$ is the state vector, $\mathbf{u} \in \mathbf{R}^{m}$ the control vector, $U$ the control set, $F(\mathbf{x})$ a continuous differentiable function; $S\left(\mathbf{x}\left(t_{f}\right)\right)$ is a set of $r$ equations, representing a terminal manifold of dimension $n-r$; the final time $t_{f}$ is given or is implicitly defined by (5).

A set of necessary conditions for such a minimax problem has been obtained in Ref. 1. For the convenience of the reader, we list briefly part of them here.

Suppose that, for an optimal pair $\mathbf{u}(t)$ and $\mathbf{x}(t), t_{0} \leq t \leq t_{f}, F(\mathbf{x}(t))$ attains its maximum $k$ times at isolated points $t_{1}, t_{2}, \ldots, t_{k}$. Then, there exists an adjoint vector $\mathbf{p}(t)$ so that the following conditions are satisfied. Define the Hamiltonian

$$
H(\mathbf{x}, \mathbf{p}, \mathbf{u})=\mathbf{p}^{T} \mathbf{f}(\mathbf{x}, \mathbf{u}) .
$$

Then,

$$
\begin{aligned}
& \dot{\mathbf{p}}(t)=-\partial H / \partial \mathbf{x}, \quad t \in\left[t_{0}, t_{1}\right),\left(t_{1}, t_{2}\right), \ldots,\left(t_{k}, t_{f}\right], \\
& H(\mathbf{x}, \mathbf{p}, \mathbf{u})=\max _{v \in U} H(\mathbf{x}, \mathbf{p}, \mathbf{v})=C, \\
& \mathbf{p}\left(t_{i}^{+}\right)=\mathbf{p}\left(t_{\mathbf{i}}^{-}\right)+\mu_{i}\left[\partial F\left(\mathbf{x}\left(t_{i}\right)\right) / \partial \mathbf{x}\right], \\
& \sum_{i=1}^{k} \mu_{i}=1, \\
& \mu_{i} \geq 0, \quad i=1,2, \ldots, k, \\
& \mathbf{p}\left(t_{f}\right)=-\left[\partial \mathbf{S}^{T}\left(\mathbf{x}_{f}\right) / \partial \mathbf{x}_{f}\right] \alpha,
\end{aligned}
$$

where $\alpha$ is an $r$-dimensional multiplier, $C$ in (7) is a constant and is equal to zero of $t_{f}$ is unspecified.

If $F(\mathbf{x}(t))$ attains its maximum value in some finite intervals $\left(t_{1}, t_{1}^{\prime}\right), \ldots,\left(t_{k}, t_{k}^{\prime}\right)$, we say that $F(\mathbf{x})$ has a flat maximum. Assume that

$$
\partial \dot{F}(\mathbf{x}(t), \mathbf{u}(t)) / \partial \mathbf{u} \neq 0, \quad \forall t \in\left(t_{i}, t_{i}^{\prime}\right), i=1, \ldots, k .
$$

For each part of the optimal trajectory $\mathbf{x}(t), t_{i} \leq t \leq t_{i}^{\prime}$, define the set $\omega_{i}(\mathbf{x}) \subset U$ as follows: $\forall \mathbf{v} \in \omega_{i}(\mathbf{x})$,

$$
\begin{aligned}
& \dot{F}(\mathbf{x}, \mathbf{v})=0, \\
& \partial \dot{F}(\mathbf{x}, \mathbf{v}) / \partial \mathbf{v} \neq 0 .
\end{aligned}
$$

For simplicity, we shall assume that $\omega_{i}(\mathbf{x})$ does not consist of the boundary points of $U$. Then, outside the intervals $\left(t_{1}, t_{1}^{\prime}\right), \ldots,\left(t_{k}, t_{k}^{\prime}\right),(6)$ and (7) still apply. Within the intervals $\left(t_{i}, t_{i}^{\prime}\right)$, we have

$$
\begin{gathered}
\dot{\mathbf{p}}(t)=-\partial H / \partial \mathbf{x}+\nu_{i}(t)[\partial \dot{F}(\mathbf{x}, \mathbf{u}) / \partial \mathbf{x}], \\
H(\mathbf{x}, \mathbf{p}, \mathbf{u})=\max _{v \in \omega_{i}(x)} H(\mathbf{x}, \mathbf{p}, \mathbf{v})=C,
\end{gathered}
$$


where $\nu_{i}(t)$ is a scalar function satisfying

$$
\begin{aligned}
& \partial H(\mathbf{x}(t), \mathbf{p}(t), \mathbf{u}(t)) / \partial \mathbf{u} \\
& =\nu_{i}(t)[\partial \dot{F}(\mathbf{x}(t), \mathbf{u}(t)) / \partial \mathbf{u}], \quad \forall t \in\left(t_{i}, t_{i}^{\prime}\right) .
\end{aligned}
$$

Furthermore (8), (9), and (11) remain applicable. The above necessary conditions will be used in Sections 4 and 5.

The minimization of the peak heating rate or the minimization of the peak deceleration during atmospheric entry has a very strong practical interest. Excellent work in this regard has been done by Miele et al. in recent years (Refs. 2-5). They have successfully obtained approximate numerical solutions to the minimization of the peak heating rate, and the results raise the interest for further exploration.

In Section 2, the heating rate and the deceleration due to aerodynamic drag are defined. Then, some properties concerning their peak values along a skip trajectory are investigated in Section 3. The minimization of the peak heating rate is considered for a coplanar aeroassisted orbital maneuver in Section 4 . The trajectory shaping is achieved by lift modulation. With the aid of the necessary conditions for minimax problems given in Ref. 1 and stated above, the property of the aerodynamic control is analyzed. In Section 5 , two numerical experiments are conducted; the analysis in the previous sections is verified. Section 6 gives a short summary.

\section{Heating Rate and Deceleration}

One of the prime concerns about the atmospheric flight of a hypervelocity vehicle such as the future National Aero-Space Plane (NASP) is the heat absorbed by the vehicle. Usually, it is sufficient to consider the heating rate. The heating rate at a particular point, for instance the stagnation point, is known to be proportional to the quantity

$$
Q=\rho^{1 / 2} V^{m},
$$

where $\rho$ is the density of the atmosphere and $V$ the speed of the point. The exponent $m$ can be taken nearly equal to 3 .

During the reentry in the atmosphere, another important consideration in manned space flight is the deceleration due to aerodynamic drag, which we call deceleration in brief. It has the following form (in $g$ 's)

$$
D=-\dot{V} / g=\rho S V^{2} C_{D} / 2 m g,
$$

where $S$ is the reference area, $m$ the mass, $C_{D}$ the drag coefficient. 
For the convenient transition between atmospheric flight and Keplerian motion of the vehicle, we shall use the modified Chapman's variables as proposed in Refs. 6 and 7,

$$
\begin{aligned}
& z=\left(\rho S C_{L}^{*} / 2 m\right) \sqrt{ }(r / \beta), \quad v=V^{2} / g r, \\
& s=\int_{0}^{t}(V / r) \cos \gamma d t .
\end{aligned}
$$

The atmosphere is assumed to be locally exponential with the inverse scale height $\beta$, that is,

$$
d \rho=-\beta \rho d r
$$

where $r$ is the radius from the center of the earth to the vehicle. The variable $z$ is proportional to $\rho$ and hence can be regarded as the replacement of the altitude with increasing $z$ for decreasing altitude. The variable $v$ is the dimensionless kinetic energy, and therefore is a measure of the speed. For $|\gamma|<90^{\circ}$, the monotonically increasing variable $s$, by its definition, is the dimensionless arc length and can be taken as independent variable. The drag polar considered is the parabolic drag polar

$$
C_{D}=C_{D 0}+K C_{L}^{2} \text {. }
$$

From the condition at maximum lift-to-drag ratio,

$$
\begin{aligned}
& C_{L}^{*}=\sqrt{ }\left(C_{D 0} / K\right), \quad C_{D}^{*}=2 C_{D 0}, \\
& E^{*}=(L / D)_{\max }=1 / 2 \sqrt{ }\left(K C_{D 0}\right),
\end{aligned}
$$

we define the normalized lift coefficient

$$
\lambda=C_{L} / C_{L}^{*} \text {. }
$$

With these definitions, we have the dimensionless equations of motion for a nonthrusting vehicle considered as a point mass,

$$
\begin{aligned}
& d z / d s=-k^{2} z \tan \gamma, \\
& d v / d x=-k z v\left(1+\lambda^{2}\right) / E^{*} \cos \gamma-(2-v) \tan \gamma, \\
& d \gamma / d s=k z \lambda \cos \sigma / \cos \gamma+(1-1 / v), \\
& d \theta / d s=\cos \psi / \cos \phi, \\
& d \phi / d s=\sin \psi, \\
& d \psi / d s=k z \lambda \sin \sigma / \cos ^{2} \gamma-\cos \psi \tan \phi .
\end{aligned}
$$

In the above equations, $\gamma$ is the flight path angle, while $\theta, \phi$, and $\psi$ are the longitude, latitude, and heading angle, respectively. The aerodynamic controls are the normalized lift coefficient $\lambda$ and the bank angle $\sigma$. The quantity $k^{2}=\beta r$ is treated as constant with the value 900 for the Earth's atmosphere. 
Using the dimensionless variables, (16) can be rescaled as

$$
Q=\sqrt{ }\left(k z v^{m}\right) \text {. }
$$

On the other hand, from (17), the deceleration corresponding to a drag coefficient $C_{D}=C_{D}^{*}$ is proportional to the following function (still designated by $D$ ), with a proportionality constant $1 / E^{*}$,

$$
D=k z v \text {. }
$$

Since $Q$ is a monotonic function of $k z v^{m}$, for the convenience of the following discussion we take $m=3$ and redefine

$$
Q=k z v^{3} \text {. }
$$

\section{Two Properties}

A skip trajectory can be the aeroassisted part of an orbital maneuver. The trajectory may be divided into descending and ascending phases, separated by the lowest altitude point on the trajectory. Along any skip trajectory, $Q$ and $D$ must have maximum values of their own which we call the peak heating rate and the peak deceleration. Regarding them, we have the following properties valid for the general case of three-dimensional maneuver with lift and bank modulation.

Property 3.1. The peak heating rate and the peak deceleration both occur in the descending phase, but not at the same instant.

This can be readily seen by differentiating (25) and (26) to obtain the conditions for stationary values of $D$ and $Q$. We have

$$
\begin{aligned}
d D / d t & =(d D / d s)(d s / d t) \\
& =k(v(d z / d s)+z(d v / d s))(d s / d t), \\
d Q / d t & =(d Q / d s)(d s / d t) \\
& =k v^{2}(v(d z / d s)+3 z(d v / d s))(d s / d t) .
\end{aligned}
$$

By definition (18), $d s / d t>0$. At the entry of the atmosphere, $d z / d s>0$ and $z \approx 0$, because the density is almost zero. Thus, $d D / d t>0$ and $d Q / d t>0$. At the lowest point of the trajectory, $d z / d s=0$ and $d v / d s<0$. Therefore, $d D / d t<0$ and $d Q / d t<0$. The maxima of $D$ and $Q$ must occur during descent. 
By simple substitution of the equations of motion (23) into (27) and (28), we arrive at

$$
\begin{aligned}
d D / d t & =-k z\left[k z v\left(1+\lambda^{2}\right) / E^{*} \cos \gamma+\left(\left(k^{2}-1\right) v+2\right) \tan \gamma\right] \\
& \times(d s / d t) \\
d Q / d t & =-k z v^{2}\left[3 k z v\left(1+\lambda^{2}\right) / E^{*} \cos \gamma+\left(\left(k^{2}-3\right) v+6\right) \tan \gamma\right] \\
& \times(d s / d t)
\end{aligned}
$$

Since $d D / d t$ and $d Q / d t$ only contain $\lambda, d D / d t$ and $d Q / d t$ should be zero at their peak value points provided that $\lambda$ is continuous there. As a result, $\gamma$ must be negative at those points for (29) and (30) to be zero, because the coefficients of $\tan \gamma$ in both equations are positive.

From (27) and (28) one can easily deduce that $d D / d t$ and $d Q / d t$ will not be zero simultaneously unless $d z / d s=d v / d s=0$ at the same time. But this generally will not be true, since $d z / d s=d v / d s=0$ indicates that the total energy of the vehicle has a stationary point, while we know that the total energy is monotonically decreasing because of the presence of aerodynamic drag.

Property 3.2. Along any skip trajectory, the peak heating rate occurs earlier than the peak deceleration does.

To show this property, first we claim that the function $z / v$ is monotonically increasing in the descending phase. This is because

$$
\begin{aligned}
(d / d s)(z / v) & =\left(z / v^{2}\right)\left[k z v\left(1+\lambda^{2}\right) / E^{*} \cos \gamma\right. \\
& \left.+\left(2-\left(k^{2}+1\right) v\right) \tan \gamma\right] .
\end{aligned}
$$

In the descending phase $\gamma<0$, and

$$
\left(2-\left(k^{2}+1\right) v\right) \leq 0, \quad \text { if } v \geq 2 /\left(k^{2}+1\right) .
$$

This means that $V \geq 0.047 V_{c}$, where $V_{c}$ is the circular speed. This lower bound approximately corresponds to a Mach number of 1.1, which is far lower than any reasonable speed along a skip trajectory. In conclusion, $d(z / v) / d s>0$ in the descending phase.

Let us consider two functions of $z$ and $v$ with the forms

$$
\begin{array}{ll}
F_{1}=k z v^{m}, & m>0, \\
F_{2}=k z v^{n}, & n>0 .
\end{array}
$$


By a similar analysis as that following Property 3.1, we are convinced that both $F_{1}$ and $F_{2}$ achieve their maxima during the descending phase. Let

$$
\begin{aligned}
& F_{1}\left(t_{1}\right)=\max _{t_{0} \leq t \leq t_{f}}\left(k z v^{m}\right)=k z_{1} v_{1}^{m}, \\
& F_{2}\left(t_{2}\right)=\max _{t_{0} \leq t \leq t_{f}}\left(k z v^{n}\right)=k z_{2} v_{2}^{n} .
\end{aligned}
$$

We will show that, if $m \geq n$, then $t_{2} \geq t_{1}$. Suppose the contrary, that is,

$$
t_{1}>t_{2} \text {. }
$$

Consequently,

$$
z_{1} / v_{1}>z_{2} / v_{2}
$$

or

$$
v_{2}>\left(z_{2} / z_{1}\right) v_{1} .
$$

For any $q \geq 0$,

$$
v_{2}^{q}>\left(z_{2}^{q} / z_{1}^{q}\right) v_{1}^{q} .
$$

By (34) and (39),

$$
k z_{1} v_{1}^{m} \geq k z_{2} v_{2}^{m}=k z_{2} v_{2}^{m-q} v_{2}^{q}>k z_{2} v_{2}^{m-q}\left(z_{2}^{q} / z_{1}^{q}\right) v_{1}^{q} .
$$

Hence,

$$
k z_{1}^{q+1} v_{1}^{m-q}>k z_{2}^{q+1} v_{2}^{m-q} .
$$

Let

$$
q=(m-n) /(n+1) \geq 0 .
$$

Equation (41) leads to

$$
\left(k z_{1} v_{1}^{n}\right)^{(q+1)}>\left(k z_{2} v_{2}^{n}\right)^{(q+1)} .
$$

Hence,

$$
k z_{1} v_{1}^{n}>k z_{2} v_{2}^{n} \text {. }
$$

But (43) contradicts (35). Hence, the assumption (36) is false and $t_{2}>t_{1}$. If we identify

$$
\begin{aligned}
& Q=F_{1}=k z v^{3}, \\
& D=F_{2}=k z v,
\end{aligned}
$$

the above arguments assert that the peak heating rate appears no later than the peak deceleration does. Property 3.1 says that they will not occur at the same time. Then, the peak heating rate must occur earlier and Property 3.2 is justified. 


\section{Minimization of the Peak Heating Rate}

In some previous work, a dual effect has been observed, that is: on a trajectory with inequality constraint on the heating rate, the peak deceleration is also reduced and vice versa (Refs. 3,5 , and 8 ). We thus concentrate on the minimization of the peak heating rate in Sections 4 and 5 .

Suppose that we consider an aeroassisted coplanar orbital maneuver. For coplanar motion, the equations of motion (23) reduce to

$$
\begin{aligned}
& d z / d s=-k^{2} z \tan \gamma, \\
& d v / d s=-k z v\left(1+\lambda^{2}\right) / E^{*} \cos \gamma-(2-v) \tan \gamma, \\
& d y / d s=k z \lambda / \cos \gamma+(1-1 / v) .
\end{aligned}
$$

The only aerodynamic control being the normalized lift coefficient $\lambda$, a practical control constraint is

$$
|\lambda| \leq \lambda_{\max } .
$$

Let

$$
\begin{aligned}
F & =Q=k z v^{3}, \\
\dot{F} & =d F / d s \\
& =-k z v^{2}\left[3 k z v\left(1+\lambda^{2}\right) / E^{*} \cos \gamma+\left(\left(k^{2}-3\right) v+6\right) \tan \gamma\right] .
\end{aligned}
$$

The objective is to minimize the maximum of (47), subject to (45) and (46) with the given boundary conditions. The final value $s_{f}$ is unspecified. By the necessary conditions for minimax problems in Section 1, we construct the Hamiltonian $H$,

$$
\begin{aligned}
H & =-p_{z} k^{2} z \tan \gamma-p_{v}\left[k z v\left(1+\lambda^{2}\right) / E^{*} \cos \gamma+(2-v) \tan \gamma\right] \\
& +p_{\gamma}[k z \lambda / \cos \gamma+(1-1 / v)]=0 .
\end{aligned}
$$

According to (7), the optimal $\lambda$ is cbtained by $\partial H / \partial \lambda=0$, provided that $\lambda$ is in the interior of control set (46). This leads to the control

$$
\lambda=E^{*} p_{\gamma} / 2 v p_{v} \text {. }
$$

The second-order optimality condition is

$$
\partial^{2} H / \partial \lambda^{2}=-2 k z v p_{v} / E^{*} \cos \gamma<0 .
$$

Since $|\gamma|<90^{\circ}$, we must have

$$
p_{v}>0 \text {. }
$$

If for certain initial and terminal conditions, $F$ attains a unique isolated maximum along an optimal minimax trajectory at $s_{1} \in\left(s_{0}, s_{f}\right)$, the adjoint 
vector $\mathbf{p}$ is subject to the discontinuity given by (8). But we will show that the control $\lambda$ is continuous at $s_{1}$, and is equal to zero if $\lambda$ is interior at $s_{1}$. At $s_{1}$, the jump conditions (8) and (9) apply and we have

$$
\begin{aligned}
& p_{z}^{+}=p_{z}^{-}+k v_{1}^{3}, \\
& p_{v}^{+}=p_{v}^{-}+3 k z_{1} v_{1}^{2}, \\
& p_{\gamma}^{+}=p_{\gamma}^{-},
\end{aligned}
$$

where in (52) $\mu_{1}=1$ from (9), since a unique maximum is assumed. From (52c), $p_{\gamma}$ is continuous. Denote $p_{\gamma}\left(s_{1}\right)$ by $p_{\gamma 1}$. By (50) and (52), we have

$$
\begin{aligned}
& \lambda^{+}=E^{*} p_{\gamma}^{+} / 2 v_{1} p_{v}^{+}=E^{*} p_{\gamma 1} /\left(2 v_{1} p_{v}^{-}+6 k z_{1} v_{1}^{3}\right), \\
& \lambda^{-}=E^{*} p_{\gamma 1} / 2 v_{1} p_{v}^{-} .
\end{aligned}
$$

Since $p_{v}^{-}>0$ by (51), it is clear from (53) and (54) that

$$
\begin{array}{ll}
\left|\lambda^{-}\right|>\left|\lambda^{+}\right|, & \text {if } p_{\gamma 1} \neq 0, \\
\lambda^{-}=\lambda^{+}=0, & \text { if } p_{\gamma 1}=0 .
\end{array}
$$

If (55) is true, then

$$
\left(\lambda^{-}\right)^{2}>\left(\lambda^{+}\right)^{2}
$$

By noticing that all the state variables are continuous at $s_{1}$ and by Property $3.1, \gamma\left(s_{1}\right)<0$, the substitution of (57) into (48) yields

$$
\dot{F}^{+}>\dot{F}^{-} \text {. }
$$

But by definition, $F\left(s_{1}\right)$ is the maximum of $F$, it is necessary that

$$
\dot{F}^{-} \geq 0, \quad \dot{F}^{+} \leq 0 .
$$

This implies that

$$
\dot{F}^{-} \geq \dot{F}^{+} \text {. }
$$

Condition (58) is contradictory to (60). Therefore, only (56) is true. The continuity of $\lambda$ at $s_{1}$ results in

$$
\dot{F}^{+}=\dot{F}^{-}=0 \text {. }
$$

The local behavior of the optimal $\lambda$, as shown in (56), can also be observed in the approximate numerical solutions to the problem in Refs. 2 and 3. It may serve as a check point for any numerical solution, and hence it indicates that the approximate solutions in Refs. 2 and 3 are sufficiently accurate. 


\section{Numerical Experiments}

Example 5.1. For the purpose of illustration of the previous analysis, we simply specify the entry and exit conditions of a skip trajectory, rather than involving more elaborate terminal constraints. First, the following conditions are chosen:

$$
\begin{array}{llll}
s_{0}=0, & z_{0}=0.0002, & v_{0}=1.3, & \gamma_{0}=-1.7^{\circ}, \\
s_{f}=\text { free }, & z_{f}=0.0002, & v_{f}=1.251, & \gamma_{f}=1.4^{\circ} .
\end{array}
$$

The initial value $z_{0}$ and final value $z_{f}$ correspond to the altitude where the atmosphere just becomes sensible. The final speed $v_{f}$ is chosen to be close to $v_{0}$, and the entry angle $\gamma_{0}$ is shallow because this example is designed to have one isolated peak heating rate along a minimax trajectory.

The vehicle is chosen with the parameters

$$
E^{*}=0.5, \quad \lambda_{\max }=2.2 .
$$

By (6), the adjoint variables satisfy the equations

$$
\begin{aligned}
\dot{p}_{z} & =-\partial H / \partial z \\
= & p_{z} k^{2} \tan \gamma+p_{v} k v\left(1+\lambda^{2}\right) / E^{*} \cos \gamma-p_{\gamma} k \lambda / \cos \gamma, \\
\dot{p}_{v}=-\partial H / \partial v & =p_{v} k z\left(1+\lambda^{2}\right) / E^{*} \cos \gamma-p_{v} \tan \gamma-p_{\gamma} / v^{2}, \\
\dot{p}_{\gamma}=-\partial H / \partial \gamma & =\left[p_{z} k^{2} z+p_{v} k z v\left(1+\lambda^{2}\right) \sin \gamma / E^{*}\right]\left(1 / \cos ^{2} \gamma\right) \\
& +\left[p_{v}(2-v)-p_{\gamma} k z \lambda \sin \gamma\right]\left(1 / \cos ^{2} \gamma\right) .
\end{aligned}
$$

The optimal $\lambda$ is determined by (7), that is,

$$
\lambda= \begin{cases}E^{*} p_{\gamma} /\left(2 v p_{v}\right), & \text { if }|\lambda| \leq \lambda_{\max }, \\ \lambda_{\max } \operatorname{sign}\left(p_{\gamma}\right), & \text { otherwise. }\end{cases}
$$

We need to integrate the systems (45) and (65), with (62) and (63) satisfied. This is a typical two-point boundary-value problem (TPBVP). We may determine $p_{v}(0)$ and $p_{\gamma}(0)$ by a shooting method; then, $\lambda(0)$ is obtained from (66) and $p_{z}(0)$ from $H=0$ [see (49)]. When $F$ attains its maximum at $s_{1}$, the jump conditions (52) apply. By the analysis in Section 4 , we should be able to see that the optimal $\lambda$ is continuous at $s_{1}$ and is equal to zero provided that $\lambda\left(s_{1}\right)$ is interior. This is the case in Fig. 1, in which the history of $\lambda$ is plotted. It starts with the maximum value 2.2 and decreases to the minimum value -2.2 , passing through zero at $s_{1}=0.1115$.

Figure 2 gives the variation of the dimensionless heating rate $\sqrt{ }\left(k z v^{3}\right)$. Its maximum value is 0.236 . In the same figure, the variation of ten times the deceleration $(10 \times k z v)$ is plotted in dashed line. The peak deceleration 


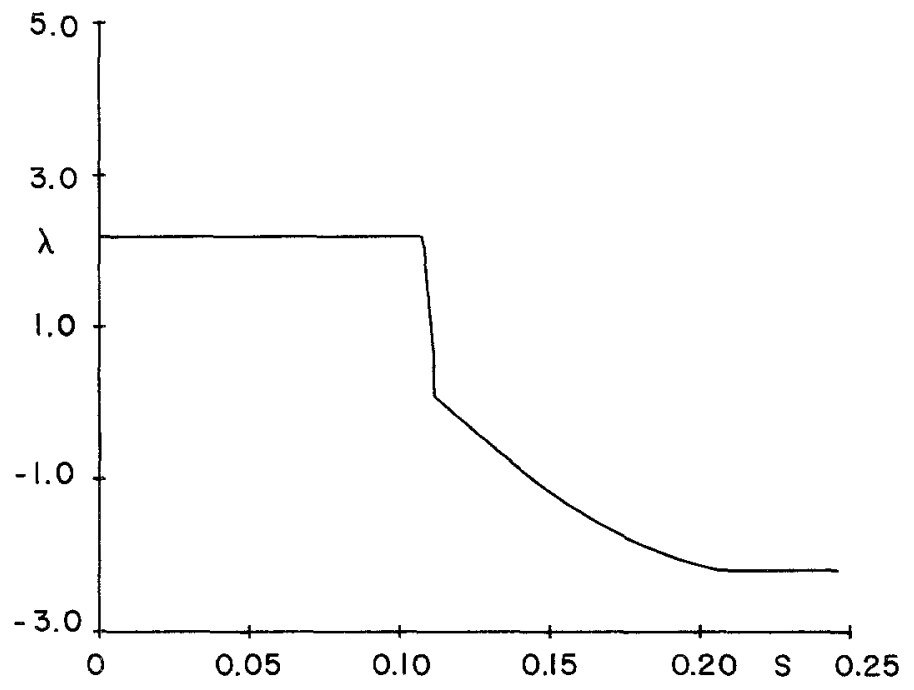

Fig. 1. Example 5.1, lift control $\lambda$.

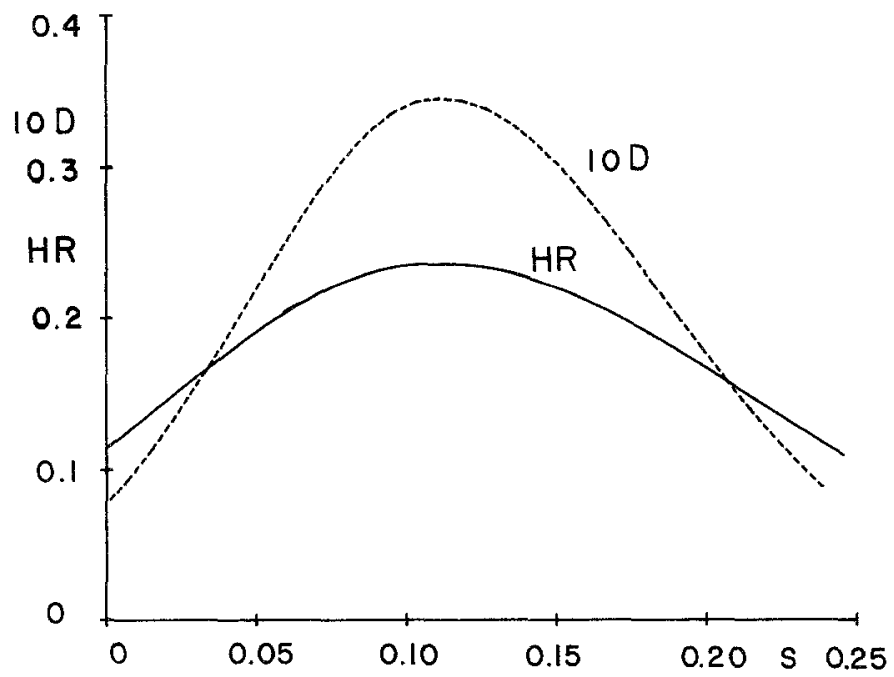

Fig. 2. Example 5.1, heating rate $H R$ and deceleration $D$. 


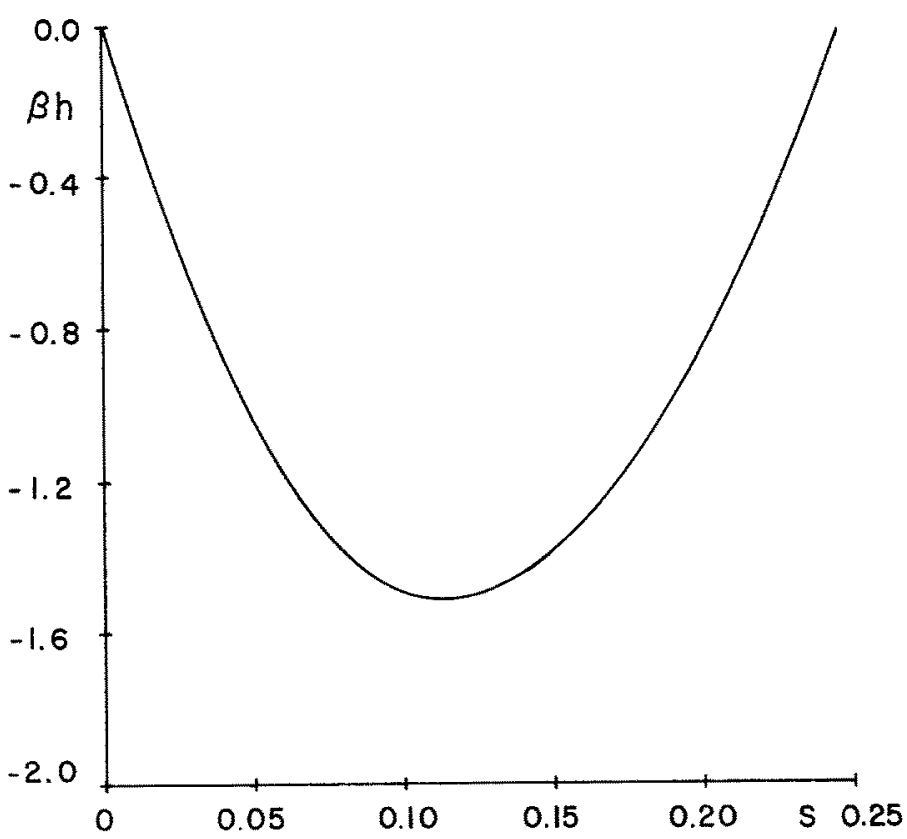

Fig. 3. Example 5.1, altitude drop $\beta h$.

is 0.035 at $s=0.1125$, occurring later than the peak heating rate, as Property 3.2 predicted. To show the optimality of the solution, we have computed a skip trajectory using constant lift coefficient. A constant value of $\lambda=-1.219$ must be selected to start with the same initial conditions (62) and have the same speed depletion. This results in a final flight path angle of $\gamma_{f}=1.6^{\circ}$ and a peak heating rate of $\max \sqrt{ }\left(k z v^{3}\right)=0.2908$.

The quantity $\ln \left(z_{0} / z\right)$ reflects the dimensionless altitude drop along the trajectory. Figure 3 shows the history of $\ln \left(z_{0} / z\right)=\beta h$. Figures 4 and 5 are the histories of the speed $v$ and flight path angle $\gamma$, respectively. It should be noticed that, since the control $\lambda$ is continuous, all the state variables have continuous first derivatives.

The above example verifies the analysis in Sections 3 and 4. But, since the vehicle does not penetrate the atmosphere deeply, the peak heating rate and the peak deceleration are less significant.

Example 5.2. Let us consider another set of boundary conditions for the same vehicle. This time, we take

$$
\begin{array}{llll}
s_{0}=0, & z_{0}=0.0002, & v_{0}=1.733, & \gamma_{0}=-5^{\circ}, \\
s_{f}=\text { free }, & z_{f}=0.0002, & v_{f}=0.94, & \gamma_{f}=5^{\circ} .
\end{array}
$$




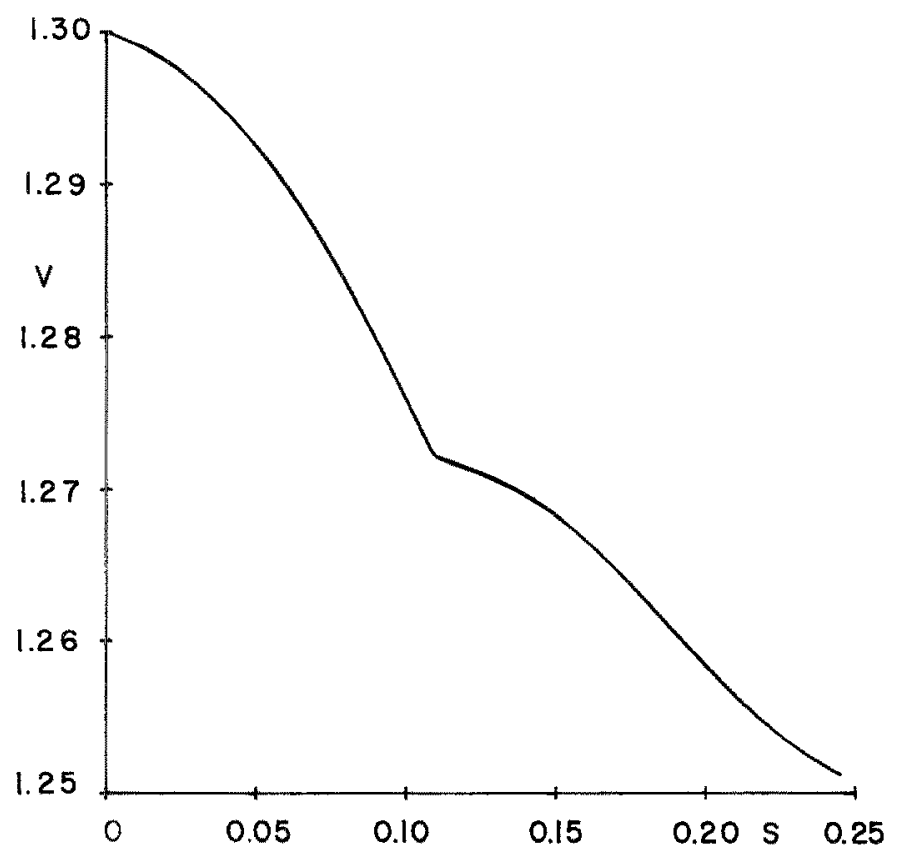

Fig. 4. Example 5.1, kinetic energy $v$

Now, the high initial speed $v_{0}$ is approximately equal to the speed which the vehicle has when returning from the geosynchronous orbit. The entry angle $\gamma_{0}$ is very steep, and the desired speed depletion is large. We naturally expect that, along the optimal trajectory, the peak heating rate is higher than in the previous case and will be attained in a finite time interval, i.e., a flat maximum.

Suppose that $F$ attains its maximum in $\left(s_{1}, s_{2}\right) \subset\left(s_{0}, s_{f}\right)$. The jump conditions (52) apply at $s_{1}$. Outside $\left(s_{1}, s_{2}\right)$, the adjoint system (65) and the control law (66) are still applicable. In $\left(s_{1}, s_{2}\right)$, the set $\omega(x)$, defined by (12), is simply determined by setting $\dot{F}=0$. We deduce that

$$
\lambda^{2}=-\left[E^{*} \sin \gamma\left(3(2-v)+k^{2} v\right)\right] / 3 k z v-1 .
$$

By (13), the adjoint state equations in $\left(s_{1}, s_{2}\right)$ are

$$
\begin{aligned}
& \dot{p}_{z}=-\partial H / \partial z+\nu(\partial \dot{F} / \partial z), \\
& \dot{p}_{v}=-\partial H / \partial v+\nu(\partial \dot{F} / \partial v), \\
& \dot{p}_{\gamma}=-\partial H / \partial \gamma+\nu(\partial \dot{F} / \partial \gamma),
\end{aligned}
$$

where by (15) $\nu$ can be solved explicitly as

$$
\nu=\left(2 p_{v} v \lambda-E^{*} p_{\gamma}\right) / 6 k z v^{3} \lambda .
$$




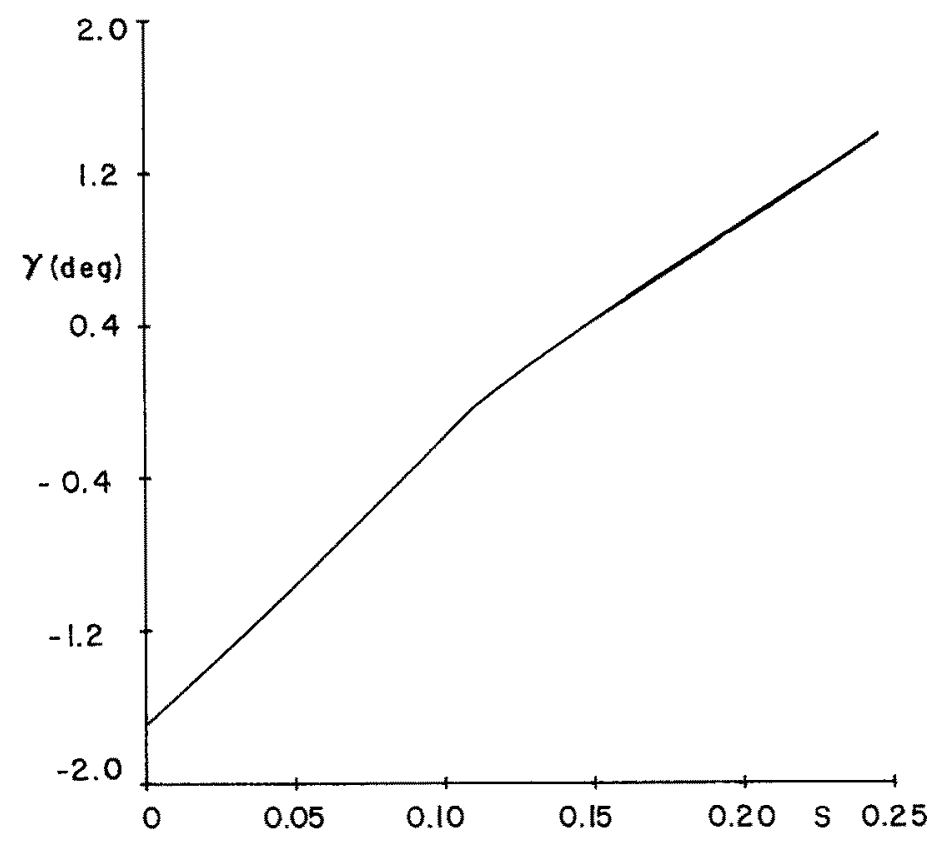

Fig. 5. Example 5.1, flight path angle $\gamma$.

We notice that, since the Hamiltonian is a quadratic function of $\lambda$, it always has a unique maximum with respect to $\lambda$, provided that $p_{v}$ and $p_{\gamma}$ do not vanish simultaneously. Hence, $H$ is regular at $s_{1}^{-}$if either or both of $p_{v}^{-}\left(s_{1}\right)$ and $p_{y}^{-}\left(s_{1}\right)$ are not zero. Then, from the discussion in Ref. 1 , we have the optimal $\lambda$ continuous at $s_{1}$, despite the discontinuity of the adjoint variables (52). Consequently, $\dot{F}\left(s_{1}^{+}\right)=\dot{F}\left(s_{1}^{-}\right)=0$.

Figure 6 shows the history of $\lambda$. It varies smoothly before the peak heating and changes rapidly afterward, with the final portion on the boundary $\lambda=\lambda_{\max }=2.2$. Notice that it is continuous at $s_{1}=0.103$ and $s_{2}=0.1077$.

The variation of the heating rate $\sqrt{ }\left(k z v^{3}\right)$ is plotted in Fig. 7. The peak heating rate, $\max \sqrt{ }\left(k z v^{3}\right)=2.33$, is attained in the interval [0.103, 0.1077]. Plotted in dashed line, in the same figure, is the variation of the deceleration $D$. Its maximum of 2.47 appears at $s=0.1142$, again occurring after the peak heating rate. A trajectory with constant lift coefficient $\lambda=0.2715$ provides the same final speed, but with a lower exit angle of $\gamma_{f}=2.5^{\circ}$. The corresponding maximum heating rate is now $\max \sqrt{ }\left(k z v^{3}\right)=2.9327$.

Figure 8 is an illustration of $\ln \left(z_{0} / z\right)$. We can see that, in this case, the vehicle dives much more deeply into the atmosphere, and hence the peak heating rate is almost ten times as large. Figures 9 and 10 are the histories of $v$ and $\gamma$, respectively. 


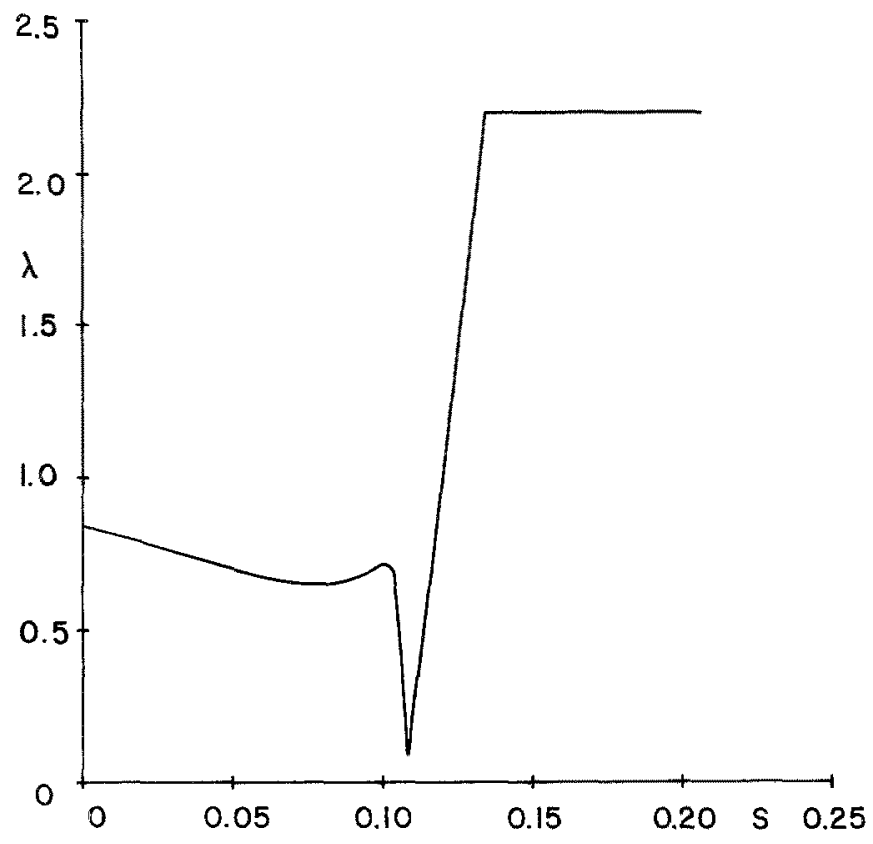

Fig. 6. Example 5.2, lift control $\lambda$.

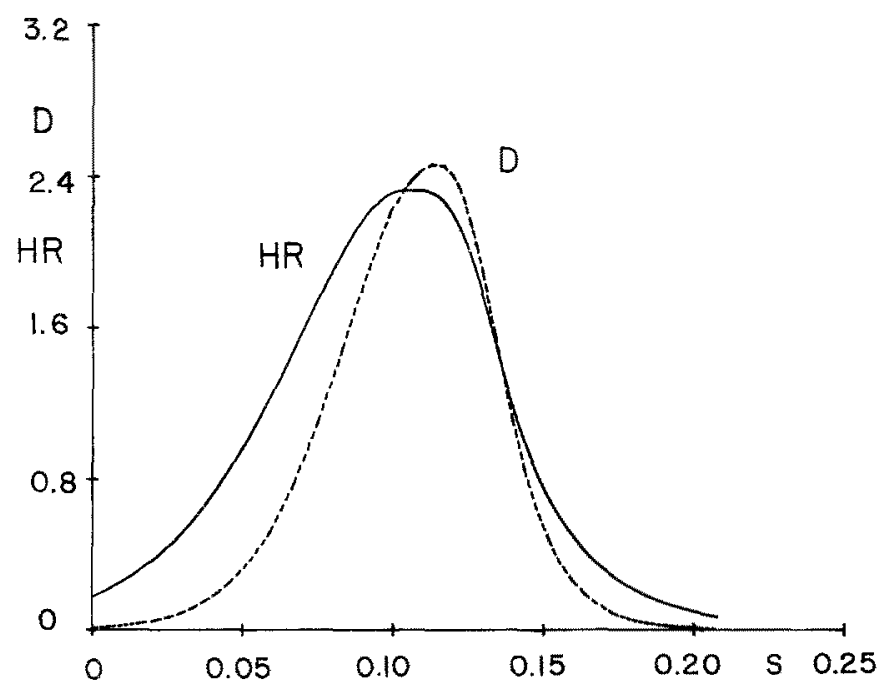

Fig. 7. Example 5.2, heating rate $H R$ and deceleration $D$. 


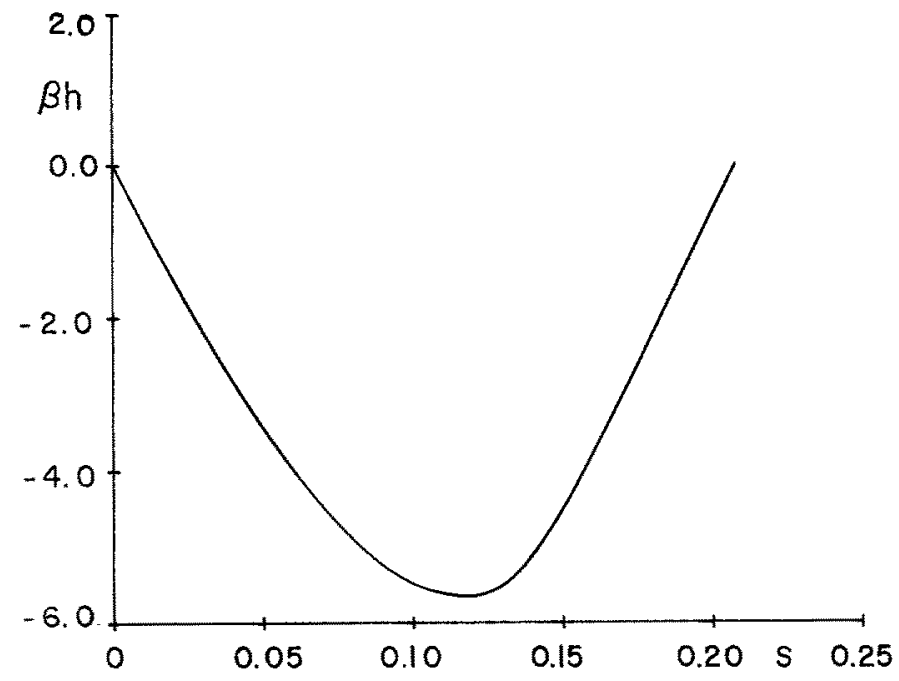

Fig. 8. Example 5.2, altitude drop $\beta h$.

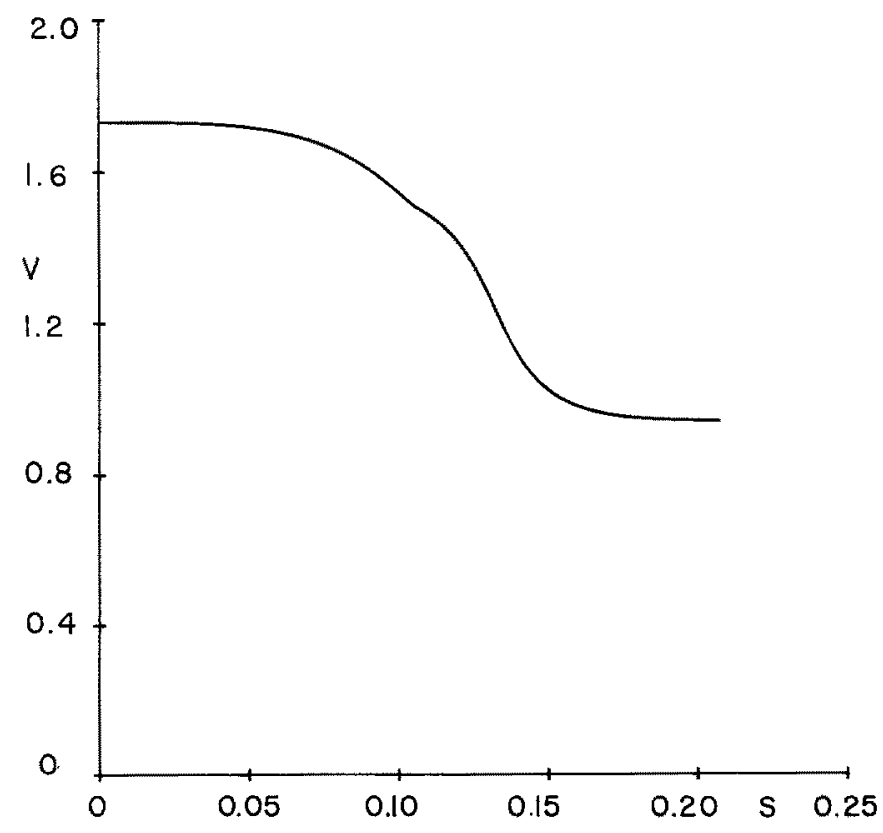

Fig. 9. Example 5.2, kinetic energy $v$. 


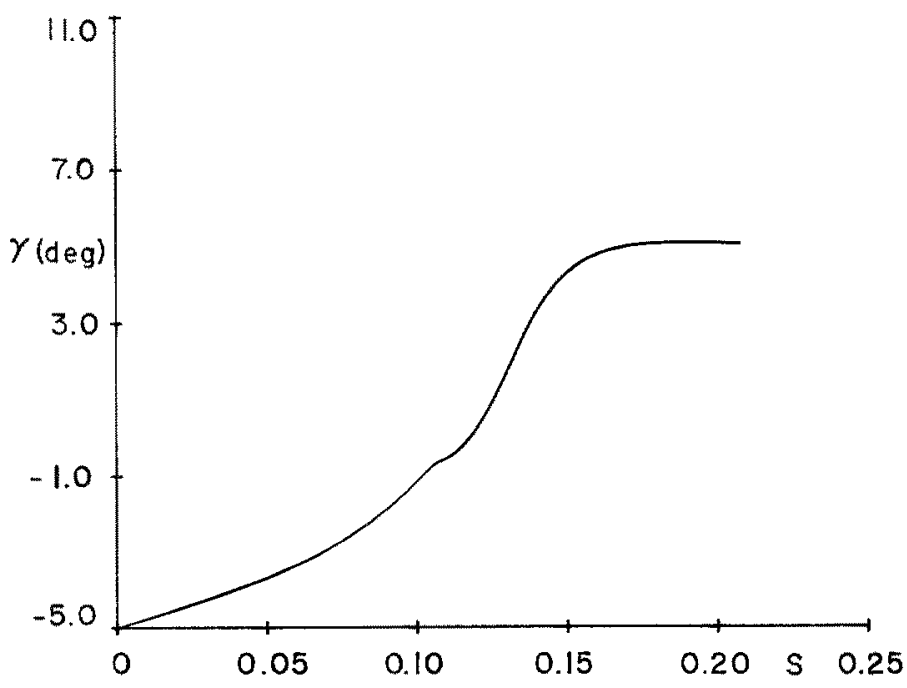

Fig. 10. Example 5.2, flight path angle $\gamma$.

\section{Conclusions}

In this paper, we have investigated some properties of the peak heating rate and the peak deceleration on a skip trajectory which can be the aeroassisted part of an orbital transfer. It is found that both occur in the descending phase of the trajectory, with the peak heating rate appearing earlier than the peak deceleration. The problem of minimization of the peak heating rate is analyzed with the aid of the necessary conditions for minimax problems from Ref. 1 . The behavior of the optimal aerodynamic control is discussed. Numerical experiments are conducted to verify the analysis and illustrate the application of the minimax technique.

\section{References}

1. VINH, N. X., and LU, P., Necessary Conditions for Maximax Problems with Application to Aeroglide of Hypervelocity Vehicles, Acta Astronautica, Vol. 15, No. 6, pp. 413-420, 1987.

2. Miele, A., and Venkataraman, P., Optimal Trajectories for Aeroassisted Orbital Transfer, Acta Astronautica, Vol. 11, Nos. 7-8, pp. 423-433, 1984.

3. Miele, A., and Basapur, V. K., Approximate Solutions to Minimax Optimal Control Problems for Aeroassisted Orbital Transfer, Acta Astronautica, Vol. 12, No. 10, pp. 809-818, 1985. 
4. Miele, A., Mohanty, B. P., Venkataraman, P, and Kuo, Y. M., Numerical Solution of Minimax Problems of Optimal Control, Part 1, Journal of Optimization Theory and Applications, Vol. 38, No. 1, pp. 97-109, 1982.

5. Miele, A., BASAPUR, V. K, and LEE, W. Y., Optimal Trajectories for Aerom assisted, Coplanar Orbital Transfer, Journal of Optimization Theory and Applications, Vol. 52, No. 1, pp. 1-24, 1987.

6. VINH, N. X., BUSEMANN, A., and CULP, R. D., Hypersonic and Planetary Entry Flight Mechanics, The University of Michigan Press, Ann Arbor, Michigan, 1980.

7. VINH, N. X., Optimal Trajectories in Atmospheric Flight, Elsevier Scientific Publishing Company, Amsterdam, Holland, 1981.

8. Chern, J. S., YANG, C. Y., Vinh, N. X., and HANSON, J. M., Deceleration and Heating Constrained Footprint of Shuttle Vehicles, Acta Astronautica, Vol. 12, No. 10, pp. 819-829, 1985. 